Article

\title{
A Hybrid Mapping Method with Position and Stiffness for Manipulator Teleoperation
}

\author{
Zainan Jiang *, Fenglei Ni, Dapeng Yang $₫$, Chongyang Li $\odot$, Fan Yang and Hong Liu
}

State Key Laboratory of Robotics and System, School of Mechatronics Engineering, Harbin Institute of Technology, Harbin 150001, China; flni@hit.edu.cn (F.N.); yangdapeng@hit.edu.cn (D.Y.); lichongyang@stu.hit.edu.cn (C.L.); yangfan_hit@163.com (F.Y.); hong.liu@hit.edu.cn (H.L.)

* Correspondence: jiangzainan@hit.edu.cn; Tel.: +86-0451-8640-2330

Received: 14 October 2019; Accepted: 18 November 2019; Published: 20 November 2019

\begin{abstract}
Transferring versatile skills of human behavior to teleoperate manipulators to execute tasks with large uncertainties is challenging in robotics. This paper proposes a hybrid mapping method with position and stiffness for manipulator teleoperation through the exoskeleton device combining with the surface electromyography (sEMG) sensors. Firstly, according to the redefinition of robot workspace, the fixed scale mapping in free space and virtual impedance mapping in fine space are presented for position teleoperation. Secondly, the stiffness at the human arm endpoint is predicted and classified into three levels based on the K nearest neighbor $(\mathrm{KNN})$ and XGBoost, and the stiffness mapping method is utilized to regulate the stiffness behavior of manipulator. Finally, the proposed method is demonstrated in three complementary experiments, namely the trajectory tracking in free space, the obstacle avoidance in fine space and the human robot interaction in contact space, which illustrate the effectiveness of the method.
\end{abstract}

Keywords: teleoperation; motion mapping; stiffness estimation; impedance control

\section{Introduction}

Over the past decades, the bilateral teleoperation systems based on feedback sensory data have been widely used in various applications such as space robots [1,2], underwater robots [3], medical robots [4], emergency response robots [5], and etc. In these systems, an operator uses a human robot interface (master) at the local site to control a robot (slave) in the remote environment to execute a task. The execution of remote task is usually assisted by feeding back the position and/or the interaction force of the slave robot, and the information of the remote environment. Although the bilateral teleoperation outperforms the traditional unilateral teleoperation, the time delay between master and slave could cause serious issues related to the system stability and reduced transparency [6], which prevent the applications of bilateral teleoperation [7]. As the bilateral teleoperation only uses the position/velocity command, the robot cannot be easily teleoperated to conduct tasks which are normally performed by humans without difficulty such as drilling, reaming, chipping and others with large uncertainty in the environment constraints.

The humans have the ability to actively adjust the impedance at their arms endpoint [8] to demonstrate a versatile and stable behavior when they perform tasks or interact with environments with large dynamic uncertainties. Electromyography (EMG) signals which describe how well the muscles respond to those signals activated by central nervous system (CNS), are considered as the best candidates to provide an insight into the overall biomechanical behavior of human arms, and have been the choice of input in many rehabilitative and human robot functionality coordinations $[9,10]$. It has been studied that the surface electromyography (sEMG) signals are highly correlated with human arm joint stiffness and the corresponding muscle tensions [11]. 
The impedance control is important for manipulator to generate compliant behavior in performing contact tasks with dynamic uncertainties [12]. Since motion trajectory transfer in traditional teleoperation systems could not generate the desired stiffness, it is difficult to enable a robot manipulator to operate in a variable compliant manner to handle the variation of tasks and environmental uncertainties [13]. However, the manipulator could have a better performance by learning human impedance featured skills, such as calligraphic writing [14], welding [15], and switching [16].

Transferring the impedance regulation of human arm endpoint to control the impedance of remote manipulators to execute tasks with large uncertainties is challenging and has been studied in recent years. Ajoudani [17] presented the concept of tele-impedance for teleoperating a KUKA manipulator stiffness based on arm stiffness estimation from the sEMG electrodes, and a ball catching experiment showed the effectiveness of the method. Yang $[18,19]$ designed an interface of a physical human robot interaction system for human impedance adaptive skill transfer, in which the limb impedance could be extracted and transferred to robots by processing the sEMG signals. Laghi [20] presented a framework of tele-impedance with force feedback under communication time delay, which could have a seamless control scheme that subsumes the performance advantages of both stability and transparency.

This paper is organized as follows. The background and related research are introduced in Section 1. In Section 2, the system overview of the hybrid mapping method for manipulator teleoperation is proposed. In Section 3, the motion mapping method for position teleoperation which includes the fixed scale mapping in free space and virtual impedance mapping in fine space is given. The stiffness mapping for compliant teleoperation is presented in Section 4 . The performance evaluation for position teleoperation and impedance teleoperation is given in Section 5. Lastly, the conclusion and future works are discussed.

\section{System Overview}

The schematic diagram of the hybrid mapping method with position and stiffness for manipulator teleoperation mainly consists of the master side and the slave side, which communicates with each other bidirectionally in real time, shown in Figure 1. The command data is transmitted by the Transmission Control Protocol (TCP) to guarantee the reliability of data transmission, and the feedback data is transmitted by the User Datagram Protocol (UDP) to obtain the low latency. With the vision and position/force feedback from the slave side, the operator utilizes the Haptic Workstation and sEMG electrodes at the master side to measure the position and stiffness at the human arm endpoint, and the hybrid mapping method with position and stiffness mapping is used to generate the position and stiffness commands of the slave robot, respectively.

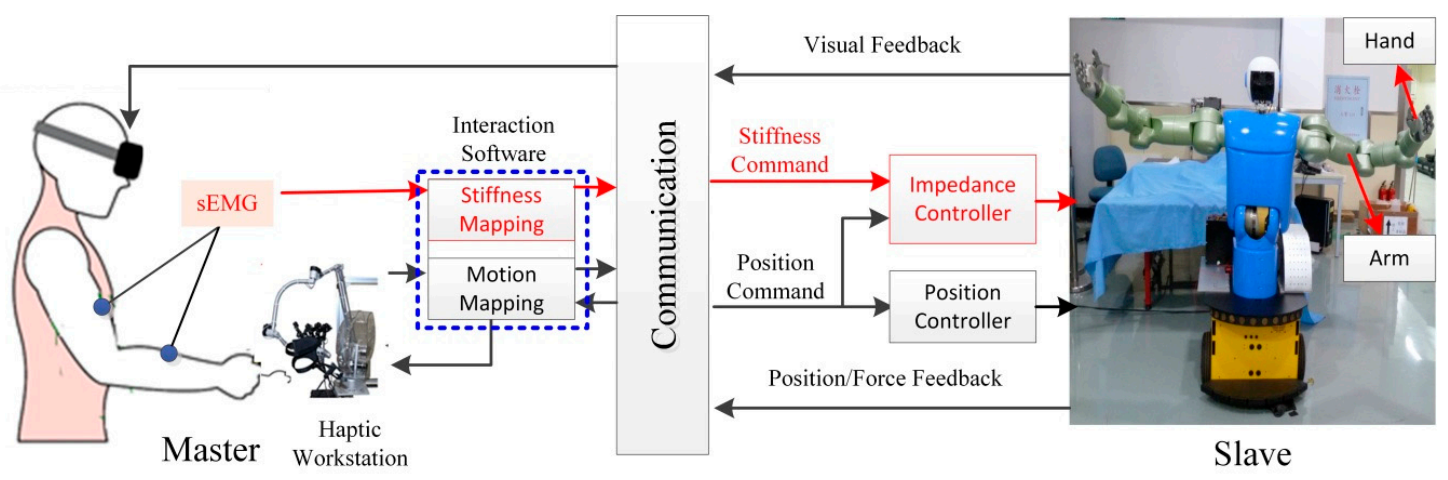

Figure 1. Schematic diagram of the hybrid mapping method for teleoperation system.

At the master side, the Haptic Workstation (CyberGlove Systems Inc., San Jose, CA, USA) consisting of dual CyberForce and dual CyberGrasp is a set of exoskeleton teleoperation device with force feedback [21]. The CyberForce is an arm exoskeleton, which tracks 6DOFs (degrees of freedom) movement of the human wrist, and provides 3DOFs force feedback along the coordinate axes directions. 
The CyberGrasp includes a data glove with 22 sensors which measure the finger joint movement of human hand, and an exoskeleton device which provides force feedback for each fingertip. The TrignoTM Wireless system (Delsys Inc., Natick, MA, USA) [22] is used to achieve the muscle electrical signals of human arm in order to predict the arm stiffness.

At the slave side, the HIT (Harbin Institute of Technology) humanoid robot consisting of an anthropomorphic upper body mounted on a wheeled mobile robot developed by HIT [23] is used as the slave robot. The robot is mainly composed of two 7DOFs light-weight manipulators, two 15DOFs five-fingered robot dexterous hands, 2DOFs robot head with the stereo vision, 2DOFs waist and 3DOFs mobile vehicle. The manipulators have position and torque sensors in each joint, and can be operated in position control mode or Cartesian impedance control mode. The dexterous hands are the HIT-DLR (German Aerospace Center) II hand, which have five identical modular fingers and a palm. In this paper, we just focus on the position and stiffness teleoperation of the manipulator.

The hybrid mapping method includes the motion mapping method for position teleoperation and stiffness mapping method for compliant teleoperation. The motion mapping method is utilized for noncontact tasks of manipulator, while the stiffness mapping method is used for contact tasks of manipulator. The two mapping methods are realized in the interaction software at the master side, and the operator can switch the methods manually through the software interface according to different situations.

\section{Motion Mapping Method for Position Teleoperation}

The objective of motion mapping for position teleoperation is to achieve ideal position trajectory tracking from the operator to the slave manipulator. Motion mapping for position teleoperation can be implemented in joint space or Cartesian space. In this paper, the Cartesian position mapping is utilized to overcome the different configuration between CyberForce and the slave manipulator.

With the motion of robot during a specific teleoperation task, the minimum distance $D_{\min }$ between the robot $(R)$ and the environment $(E)$, the target $(T)$, and the obstacles $(\mathrm{O})$ is changing over the time, which can be defined by Equation (1):

$$
D_{\text {min }}=\min (\|R-E\|,\|R-T\|,\|R-O\|)
$$

According to the minimum distance $D_{\min }$, the workspace of robot WS can be redefined into free space for fast motion, fine space for slow motion and contact space for compliant motion, shown in Figure 2. The redefined workspace WS can be expressed by Equation (2):

$$
W S= \begin{cases}\text { Free space } & D_{\min } \geq D_{1} \\ \text { Fine space } & D_{2}<D_{\min }<D_{1} \\ \text { Contact space } & D_{\min } \leq D_{2}\end{cases}
$$

where, $D_{1}$ and $D_{2}$ represent the predefined constant distance thresholds, and $D_{2}$ is usually close to zero.

Based on this redefined workspace of robot WS, different mapping methods are proposed in this paper to teleoperate the slave manipulator, and these methods are switched according to the real workspace of robot. 


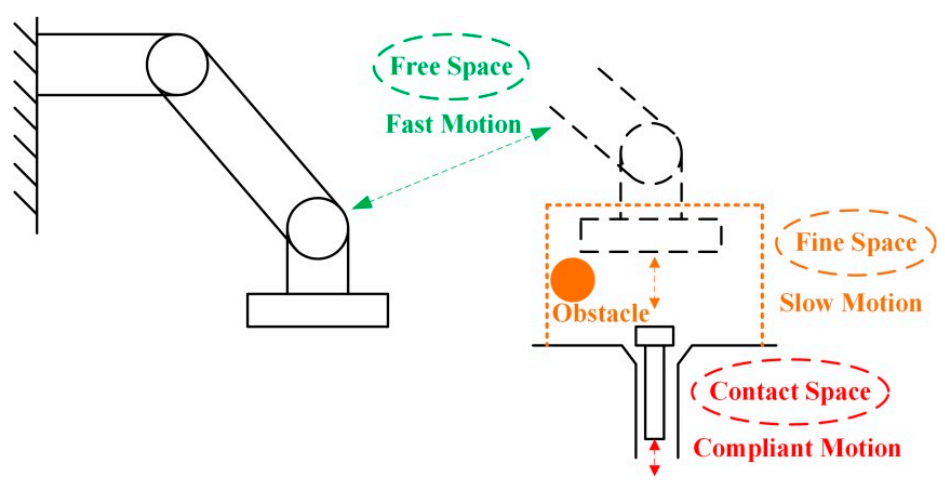

Figure 2. Workspace redefinition of robot.

\subsection{Fixed Scale Mapping in Free Space}

In the free space, the slave manipulator should be controlled to track the position at the human arm endpoint quickly and exactly. While there is no contact force in free space, the CyberForce without force feedback is used to track the motion of human arm. Considering the different configuration between human arm and slave manipulator, the appropriate mapping method should be implemented to maintain the tracking performance. For simplicity, the most frequently used fixed scale mapping method is utilized for position teleoperation in free space in this paper, as shown in Equation (3):

$$
\mathbf{X}_{\mathbf{s}}=\mathbf{K}_{\mathbf{m}} \mathbf{X}_{\mathbf{m}}+\mathbf{X}_{0}
$$

where $\mathbf{X}_{\mathbf{s}}$ is the Cartesian position vector of the slave manipulator, $\mathbf{X}_{\mathbf{m}}$ is the position vector at the operator arm endpoint obtained by CyberForce, $\mathbf{K}_{\mathbf{m}}$ is the fixed scale matrix, and $\mathbf{X}_{0}$ is the position offset vector. The detail calculation of $\mathbf{K}_{\mathbf{m}}$ and $\mathbf{X}_{0}$ can be found in [24].

\subsection{Virtual Impedance Mapping in Fine Space}

When the operator teleoperates the slave manipulator in fine space, he should guarantee the safety of whole system. That is to say, the slave manipulator should be controlled to avoid the collisions between the manipulator and the environment, the manipulated target, or the obstacles. In this case, since there is a possibility of collision, the CyberForce with force feedback is utilized in fine space to maintain the safety. To meet this requirement, the virtual impedance mapping method is proposed in this paper to obtain the safe motion in the fine space.

If there is an obstacle which can be detected by the stereo vision or prior knowledge in the remote environment, a corresponding virtual obstacle expressed with a bounding sphere can be established at the master side by the inverse process of Equation (3), shown in Figure 3. Moreover, a safe boundary sphere is used to enlarge the virtual obstacle to improve the system safety.

The Euclidean distance $\boldsymbol{d}$ between the input position $\boldsymbol{P}$ and the virtual contact boundary $\boldsymbol{P}_{S b}$ (Figure 4) at the master side is defined in Equation (4):

$$
\boldsymbol{d}=\left\|\boldsymbol{P}-\boldsymbol{P}_{S b}\right\|
$$

To improve the motion safety in fine space, the Cartesian impedance controller is utilized for the master, which can be expressed as Equation (5):

$$
\begin{gathered}
\mathbf{M}_{\mathrm{d}} \ddot{\xi}+\mathbf{B}_{\mathrm{d}} \dot{\xi}+\mathbf{K}_{\mathrm{d}} \xi=F_{\mathrm{d}} \\
\boldsymbol{F}_{\mathrm{d}}=k \boldsymbol{d}
\end{gathered}
$$


where $\mathbf{M}_{\mathrm{d}}, \mathbf{B}_{\mathrm{d}}, \mathbf{K}_{\mathrm{d}}$ are the desired inertia, viscosity and stiffness matrices of the virtual impedance controller for the master, $\boldsymbol{F}_{\mathrm{d}}$ is the desired Cartesian force, $\boldsymbol{\xi}$ is the correction term for virtual impedance controller and $k$ is the force scale factor.

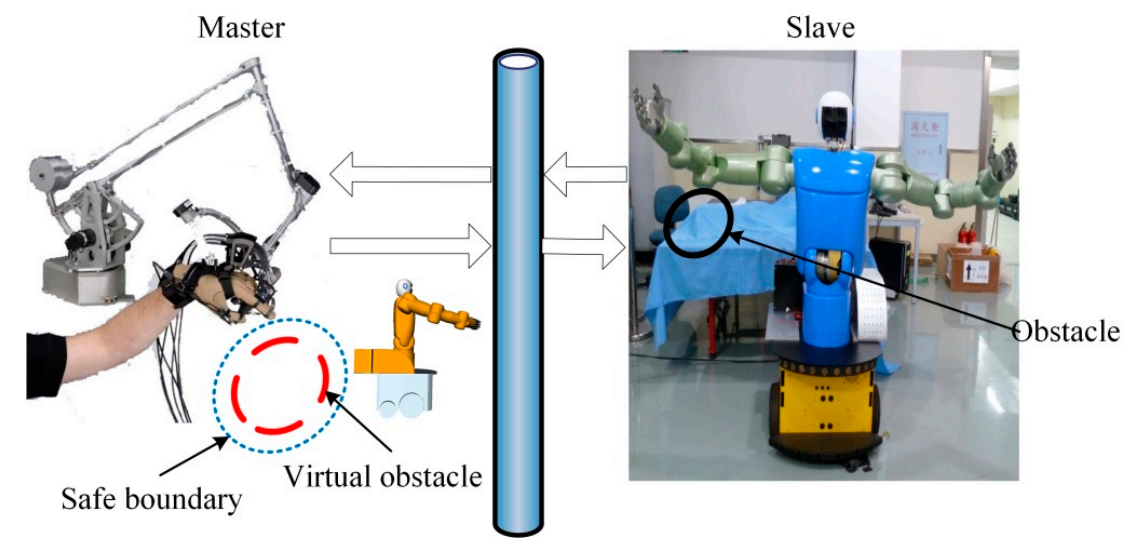

Figure 3. Diagram of virtual impedance mapping.

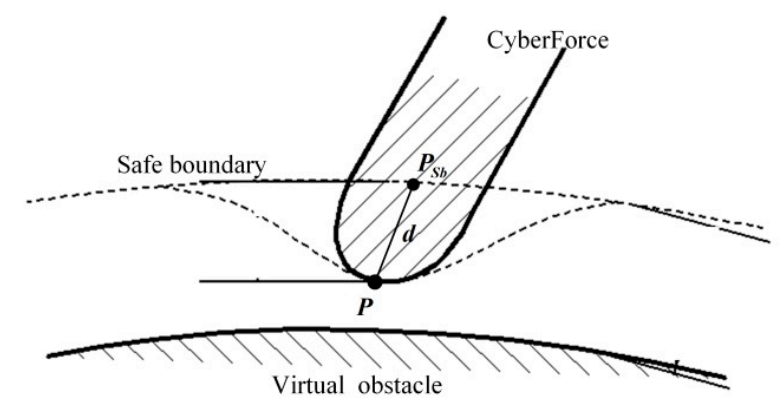

Figure 4. Distance of virtual obstacle.

Therefore, the virtual impedance mapping for robot position teleoperation in fine space can be expressed as:

$$
\mathbf{X}_{\mathbf{s}}=\mathbf{K}_{\mathbf{m}}\left(\mathbf{X}_{\mathbf{m}}-\xi\right)+\mathbf{X}_{0}
$$

The switch between the fixed scale mapping method and the virtual impedance mapping method can be achieved automatically according to the real workspace of robot. The switch is stable because the trajectories of manipulator with different mapping methods are continuous when the minimum distance of robot is around $D_{1}$. The derivation is as follows.

When the minimum distance of robot $D_{\min }$ equals to $D_{1}$, we can obtain $\boldsymbol{d}=0$ according to Equation (4). Therefore, Equation (5) can be rewritten as:

$$
\mathbf{M}_{\mathrm{d}} \ddot{\boldsymbol{\xi}}+\mathbf{B}_{\mathrm{d}} \dot{\xi}+\mathbf{K}_{\mathrm{d}} \xi=\boldsymbol{F}_{\mathrm{d}}=k \boldsymbol{d}=0
$$

Then, we can calculate $\xi=0$. After substituting this into Equation (7), we can obtain:

$$
\mathbf{X}_{\mathbf{s}_{-} D_{1}}=\mathbf{K}_{\mathbf{m}}\left(\mathbf{X}_{\mathbf{m} \_D_{1}}-0\right)+\mathbf{X}_{0}=\mathbf{K}_{\mathbf{m}} \mathbf{X}_{\mathbf{m}_{-} D_{1}}+\mathbf{X}_{0}
$$

where $\mathbf{X}_{\mathbf{m}_{-} D_{1}}$ and $\mathbf{X}_{\mathbf{s}_{-} D_{1}}$ represent the Cartesian position vector of the master and slave manipulator when the minimum distance of robot $D_{\min }$ equals to $D_{1}$.

The expression of Equation (9) is the same as Equation (3) when the minimum distance of robot $D_{\min }$ equals to $D_{1}$, which shows that the trajectories of manipulator are continuous and the switch between the fixed scale mapping method and the virtual impedance mapping method is stable. 


\section{Stiffness Mapping Method for Compliant Teleoperation}

When the minimum distance of robot $D_{\min }$ is around $D_{2}$, the velocity of the manipulator is around zero according to Equation (7) with the virtual impedance mapping method. In this situation, the operator switches to the stiffness mapping method manually through the software interface for contact tasks to achieve variable stiffness behavior of manipulator. In order to maintain the stable switch, the value of $\xi$ should be recorded and used for motion mapping during the contact tasks.

\subsection{Stiffness Model at the Human Arm Endpoint}

In order to predict the stiffness at the human arm endpoint, starting with the muscle control of a single human arm joint, the motion control biomechanical model of a single joint is established. Then, the model of force and stiffness in Cartesian space is calculated. Generally, the motion of human joint is controlled by at least two muscles, which are called the extensor and the flexor. The extensor is activated to stretch the joint while the flexor is activated for flexion, as shown in Figure 5.

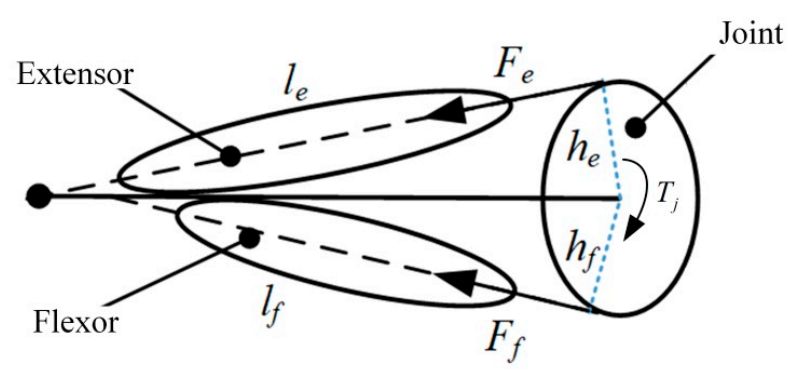

Figure 5. Muscle control model of a single joint.

Once the joint muscles receive motion commands from CNS through neurons, they are activated to generate the muscle force, which results in the moment $T_{j}$ and stiffness $K_{j}$ at the human arm joint. They can be obtained in Equations (10) and (11):

$$
\begin{gathered}
T_{j}=F_{f} h_{f}-F_{e} h_{e} \\
K_{j}=K_{f}+K_{e}=\frac{d F_{f}}{d l_{f}}+\frac{d F_{e}}{d l_{e}}
\end{gathered}
$$

where $F_{f}$ and $F_{e}$ represent the muscle forces of the flexor and extensor, $h_{f}$ and $h_{e}$ represent the moment arm of the flexor and extensor, $K_{f}$ and $K_{e}$ represent the stiffness of the flexor and extensor.

The motion at the human arm endpoint is achieved by the multi-joints motion. Therefore, the force vector $\boldsymbol{F}_{d}$ and stiffness matrix $\boldsymbol{K}_{d}$ at the human arm endpoint can be calculated in Equations (12) and (13):

$$
\begin{gathered}
\boldsymbol{F}_{\mathrm{d}}=\boldsymbol{J}^{-T}(q) \tau_{\boldsymbol{q}} \\
\boldsymbol{K}_{d}=\frac{d \boldsymbol{F}_{d}}{d \boldsymbol{x}}=\frac{d\left(\boldsymbol{J}^{-T}(q) \tau_{q}\right)}{d \boldsymbol{x}}=\frac{d\left(\boldsymbol{J}^{-T}(q)\right)}{d \boldsymbol{x}} \tau_{q}+\boldsymbol{J}^{-T}(q) \frac{d\left(\boldsymbol{\tau}_{\boldsymbol{q}}\right)}{d \boldsymbol{x}}
\end{gathered}
$$

where $\tau_{q}$ is the moment vector of human arm joints, and $J(q)$ is the Jacobian matrix of human arm.

Especially, $\boldsymbol{J}(q)$ changes very small if the joint displacement $q$ changes little, then $d\left(J^{-T}(q)\right)$ can be neglected. Therefore, the stiffness matrix $K_{d}$ at the human arm endpoint in Equation (13) can be simplified as:

$$
\boldsymbol{K}_{d}=\boldsymbol{J}^{-T}(q) \frac{d\left(\tau_{q}\right)}{d x}=J^{-T}(q) \frac{d\left(\tau_{q}\right)}{d q} \frac{d q}{d x}=J^{-T}(q) \boldsymbol{K}_{q} J^{-1}(q)
$$

where $K_{q}$ is the diagonal stiffness matrix of human arm joints. 


\subsection{Stiffness Identification Algorithm at the Human Arm Endpoint}

The force fluctuations and impedance adjustments at the human arm endpoint can be regarded as internal force regulations exerted by group of extensor and flexor muscles. Based on the high correlation between sEMG signals and generated muscle tension, there is an approximate linear relationship between the force/stiffness and the sEMG signals of the human arm, which can be described as follows:

$$
\left[\begin{array}{l}
\boldsymbol{F}_{\text {end }} \\
\boldsymbol{K}_{\text {end }}
\end{array}\right]=\left[\begin{array}{l}
\boldsymbol{T}_{F} \\
\boldsymbol{T}_{K}
\end{array}\right] \boldsymbol{P}_{\text {se }}+\left[\begin{array}{l}
0 \\
\boldsymbol{K}_{0}
\end{array}\right]
$$

where $\boldsymbol{F}_{\text {end }}$ and $\boldsymbol{K}_{\text {end }}$ are the force vector and the stiffness vector at the human arm endpoint, $\boldsymbol{P}_{s e}$ is the processed sEMG signals, $\boldsymbol{T}_{F}$ and $\boldsymbol{T}_{K}$ are the mapping matrices from the sEMG signals $\boldsymbol{P}_{s e}$ to the force vector $\boldsymbol{F}_{\text {end }}$ and the stiffness vector $\boldsymbol{K}_{\text {end }}$, and $\boldsymbol{K}_{0}$ is the stiffness offset at the human arm endpoint.

The force mapping matrix $\boldsymbol{T}_{F}$ can be obtained by the regression analysis model. However, the stiffness mapping matrix $\boldsymbol{T}_{K}$ is difficult to identify accurately. Relevant research [8] shows that the force $\boldsymbol{F}_{\text {end }}$ and the stiffness $\boldsymbol{K}_{\text {end }}$ at the human arm endpoint are decoupled if the motion and force at the human arm endpoint change little. That is to say, the muscular activities can be decomposed and mapped into two subspaces. The first mapping subspace corresponds to the force fluctuations and the second will be defined as the kernel of the former which correlates of stiffness regulations without causing any effect on generated joint/endpoint forces. Therefore, the sEMG signals $\boldsymbol{P}_{s e}$ can be decomposed into the force mapping subspace $\boldsymbol{P}_{F}$ and the force mapping null space $\boldsymbol{P}_{K}$.

$$
\boldsymbol{P}_{s e}=\boldsymbol{P}_{F} \oplus \boldsymbol{P}_{K}
$$

Then we can obtain:

$$
\boldsymbol{P}_{s e}=\mathbf{T}_{\mathbf{F}}^{-1} \mathbf{T}_{\mathbf{F}} \boldsymbol{P}_{s e}+\left(\mathbf{I}-\mathbf{T}_{\mathbf{F}}^{-1} \mathbf{T}_{\mathbf{F}}\right) \boldsymbol{P}_{s e}=\boldsymbol{P}_{F}+\boldsymbol{P}_{K}
$$

In order to obtain the relationship between $\boldsymbol{P}_{K}$ and $\boldsymbol{P}_{s e}$, we define $\boldsymbol{N}_{F}^{+}$as the kernel of the force mapping matrix $\boldsymbol{T}_{F}$.

$$
N_{F}^{+}=\mathbf{I}-\mathbf{T}_{\mathbf{F}}^{\mathbf{T}}\left(\mathbf{T}_{\mathbf{F}} \mathbf{T}_{\mathbf{F}}^{\mathbf{T}}\right)^{-1} \mathbf{T}_{\mathbf{F}}
$$

Then $\boldsymbol{P}_{K}$ can be expressed as:

$$
\boldsymbol{P}_{K}=\boldsymbol{N}_{F}^{+} \boldsymbol{P}_{\text {se }}
$$

The stiffness at the human arm endpoint can be rewritten as follows:

$$
\boldsymbol{K}_{\text {end }}=\boldsymbol{K}_{0}+\psi \boldsymbol{P}_{K}=\boldsymbol{K}_{0}+\psi \boldsymbol{N}_{F}^{+} \boldsymbol{P}_{\text {se }}
$$

where $\psi$ is the mapping matrix from the force mapping null space $\boldsymbol{P}_{K}$ to the stiffness at the human arm endpoint $\boldsymbol{P}_{\text {end }}$.

\subsection{Stiffness Identification System at the Human Arm Endpoint}

The stiffness identification experimental system at the human arm endpoint consists of three modules: the KUKA iiwa robot module (KUKA, Germany), the JR3 six-axis force sensor module (JR3 Inc., Canada) and the TrignoTM Wireless sEMG system module (Delsys Inc., USA), as shown in Figure 6. The robot module measures the displacement at the human arm endpoint caused by the random disturbance. The six-axis force sensor JR3 is used to detect the force applied by the operator hand. The sEMG electrodes on the operator arm detect the muscle electrical signals of human arm while the operator demonstrates different behaviors with different stiffness. In the experiment for stiffness identification, the operator holds the interface handle mounted at the end of Kuka iiwa, and applies appropriate force to cause the robot to move within a small scope. With the measured 
displacement, force and sEMG signals, the stiffness mapping matrix at the human arm endpoint can be identified.

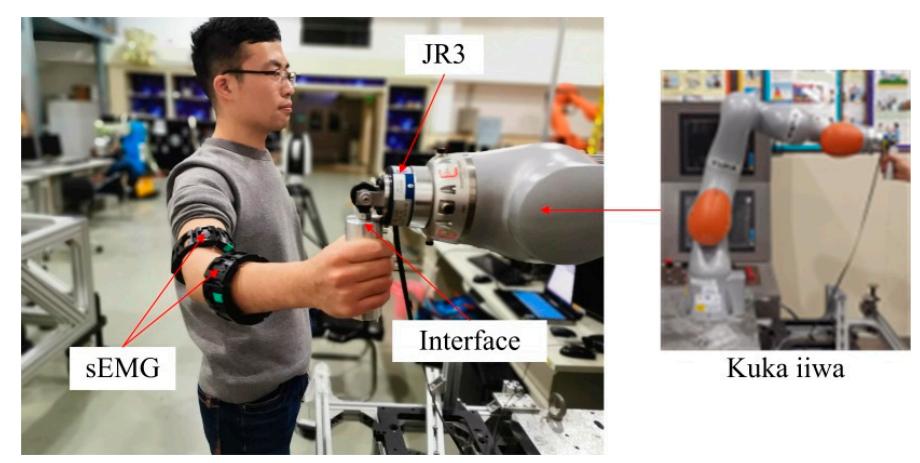

Figure 6. Experimental system of stiffness identification.

\subsubsection{Feature Extraction of sEMG Signals}

The feature extraction of sEMG signals is an important item in the stiffness identification of human arm. In this paper, the following seven features of sEMG signals are extracted. The $x_{i}$ is the $i$ th channel value of sEMG signals, $N$ is the window length of time domain, SVD represents the singular value decomposition, and WPT represents the wavelet packet transform.

(1) Modified absolute mean type1 (MAV1).

$$
\begin{aligned}
& M A V 1=\frac{1}{N} \sum_{i=1}^{N} w_{i}\left|x_{i}\right| \\
& w_{i}=\left\{\begin{array}{cc}
1, & 0.25 N \leq i \leq 0.75 N \\
0.5, & \text { other }
\end{array}\right.
\end{aligned}
$$

(2) Zero crossing point (ZC).

$$
\begin{aligned}
& \mathrm{ZC}=\sum_{i=1}^{N-1}\left[f\left(x_{i} x_{i+1}\right) \cap\left|x_{i}-x_{i+1}\right| \geq \text { threshold }\right] \\
& f(x)=\left\{\begin{array}{lc}
1, & x \geq \text { threshold } \\
0, & \text { others }
\end{array}\right.
\end{aligned}
$$

(3) Symbol change slope (SSC).

$$
\begin{aligned}
S S C & =\sum_{i=2}^{N-1}\left[f\left[\left(x_{i}-x_{i-1}\right)\left(x_{i}-x_{i+1}\right)\right]\right] \\
f(x) & =\left\{\begin{array}{l}
1, x \geq \text { threshold } \\
0, \text { others }
\end{array}\right.
\end{aligned}
$$

(4) Waveform length (WL).

$$
W L=\sum_{i=1}^{N-1}\left|x_{i+1}-x_{i}\right|
$$

(5) TD4 (MAV+ZC+SSC+WL), is the combination of MAV, ZC, SSC, and WL.

(6) Singular Value Decomposition of Wavelet Coefficient (WTSVD).

$$
W T S V D=S V D(W T(x))
$$

(7) Singular Value Decomposition of Wavelet Packet Coefficient (WPTSVD).

$$
W P T S V D=S V D(W P T(x))
$$




\subsubsection{Stiffness Classification at the Human Arm Endpoint}

For teleoperating the stiffness of the slave robot to execute most tasks, it is not necessary to adjust the end stiffness continuously and accurately. For the offline samples, the pattern classification with $\mathrm{K}$-means++ clustering is firstly used to cluster the continuous stiffness into three types. For the online applications, the K nearest neighbor (KNN) and XGBoost (eXtreme Gradient Boosting) are utilized to classify the stiffness at the human arm endpoint.

(1) Stiffness Clustering for Offline Samples

Because it is not necessary to adjust the stiffness at the robot endpoint continuously and accurately in most tasks, according to the features of sEMG signals, the pattern classification method with $\mathrm{K}$-means++ clustering is utilized to classify the stiffness $K_{\mathrm{x}}, K_{y}, K_{z}$ for offline samples at the human arm endpoint into three categories (low, middle and high). The stiffness cluster center at the human arm endpoint with K-means++ is shown in Table 1.

Table 1. Stiffness cluster center with K-means++.

\begin{tabular}{cccc}
\hline Item & Low $(\mathbf{N m})$ & Middle(Nm) & High(Nm) \\
\hline$K_{\mathrm{x}}$ & 11 & 151 & 400 \\
$K_{y}$ & 17 & 161 & 387 \\
$K_{z}$ & 16 & 126 & 416 \\
\hline
\end{tabular}

(2) Stiffness Classification for Online Applications

According to the stiffness clustering, the K nearest neighbor $(\mathrm{KNN})$ and XGBoost (eXtreme Gradient Boosting) are utilized for online applications to classify the real-time stiffness at the human arm endpoint. The features of sEMG signals are the input, and the classification results of stiffness at the human arm endpoint based on k-means++ cluster are used as output.

(a) KNN

$\mathrm{KNN}$ is a simple and widely used classification method. If most of the $\mathrm{k}$ neighbor samples in the feature space belong to a certain category, the sample also belongs to this category and has the characteristics of the samples in this category. Obviously, the parameter $\mathrm{k}$ is important for the classification effect of the model, and it is general set to less than 30. According to the actual prediction accuracy, the $\mathrm{k}$ for $K_{x}, K_{y}$ and $K_{z}$ classifiers are set to 26,18 and 22, respectively. The accuracy of stiffness classification with KNN is shown as Figure 7, and the confusion matrix of stiffness classification based on MAV1 is shown as Figure 8.

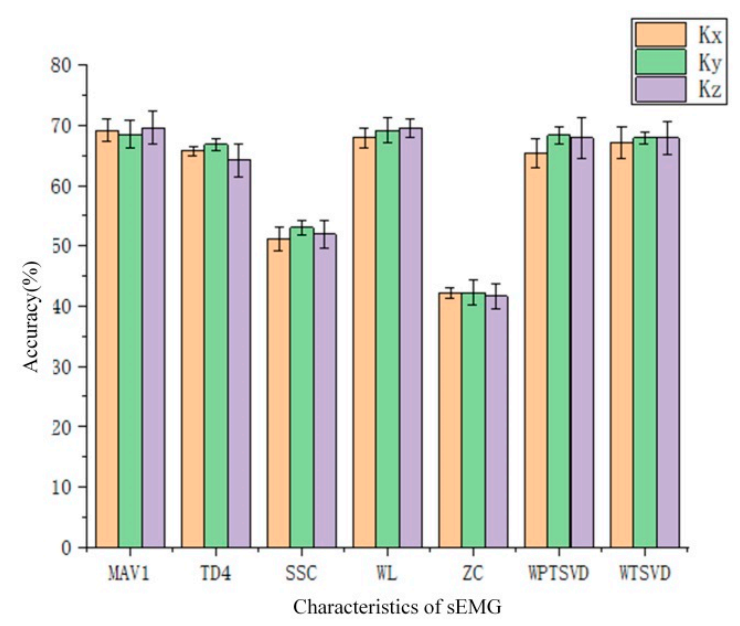

Figure 7. Accuracy of stiffness classification with KNN. 

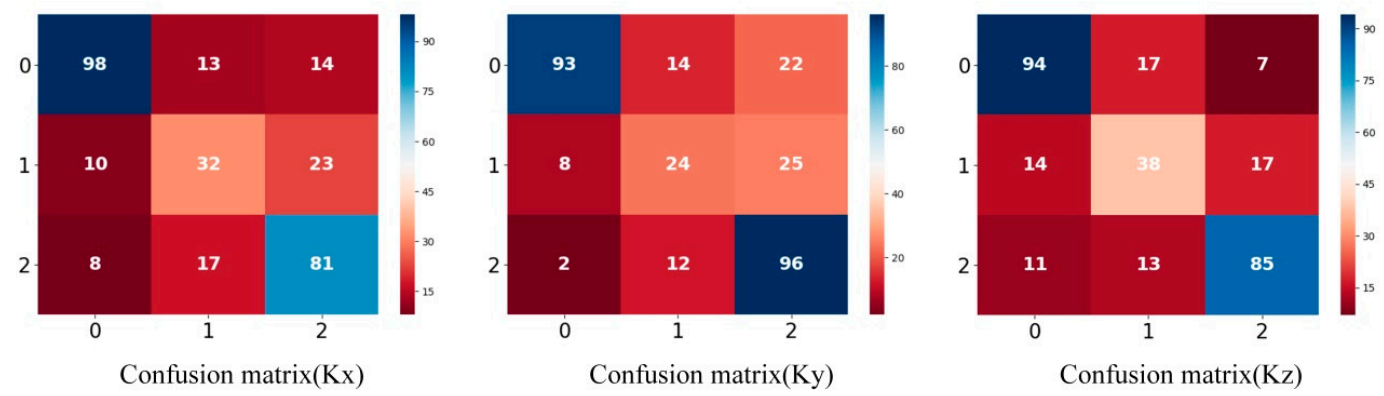

Figure 8. Confusion matrix of stiffness based on MAV1 $(0=$ high stiffness, $1=$ middle stiffness, $2=$ low stiffness).

In Figure 7, the accuracy of stiffness classification at the human arm endpoint is close to $70 \%$ when the sEMG features are extracted by MAV1, WL, WPTSVD and WTSVD, while it is only about $40 \%$ when the ZC is selected as the sEMG features. In Figure 8, it can be seen that the stiffness at the human arm endpoint in all directions has certain coupling. Moreover, the stiffness classification method with KNN is better for low stiffness and high stiffness, but worse for middle stiffness. The main reasons for this phenomenon are that the operator is difficult to determine to apply the appropriate force to generate a middle stiffness. Because of time consuming with WPTSVD, three kinds of sEMG feature (MAV1, WL and WTSVD) are used to predict the stiffness at the human arm endpoint in real-time based on KNN.

(b) XGBoost

XGBoost implements machine learning algorithms under the Gradient Boosting framework, and it is an optimized distributed gradient boosting library designed to be highly efficient, flexible and portable. In this paper, the Python package xgboost [25] is used for the stiffness classification at the human arm endpoint in online applications. Table 2 is the hyper parameter table obtained by the cross-validation. The accuracy of stiffness classification with XGBoost is shown as Figure 9, and the confusion matrix of stiffness classification based on MAV1 is shown as Figure 10.

Table 2. Hyper parameters of XGBoost (Kx, Ky, Kz).

\begin{tabular}{cccc}
\hline Item & Value & Item & Value \\
\hline learning_rate & $0.1 / 0.1 / 0.1$ & max_depth & $8 / 10 / 8$ \\
n_estimators & $80 / 45 / 85$ & min_child_weight & $3 / 1 / 0.3$ \\
subsample & $1 / 0.8 / 0.7$ & gamma & $0.4 / 0.1 / 0$ \\
colsample_bytree & $0.8 / 0.8 / 0.8$ & reg_lambda & $1.5 / 1 / 2$ \\
\hline
\end{tabular}

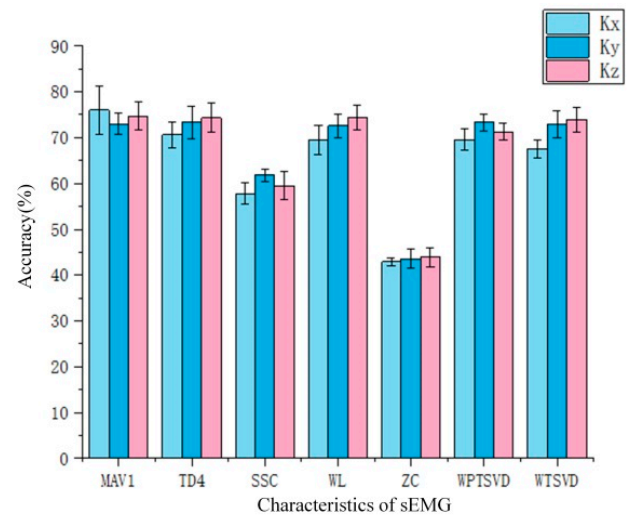

Figure 9. Accuracy of stiffness classification with XGBoost. 

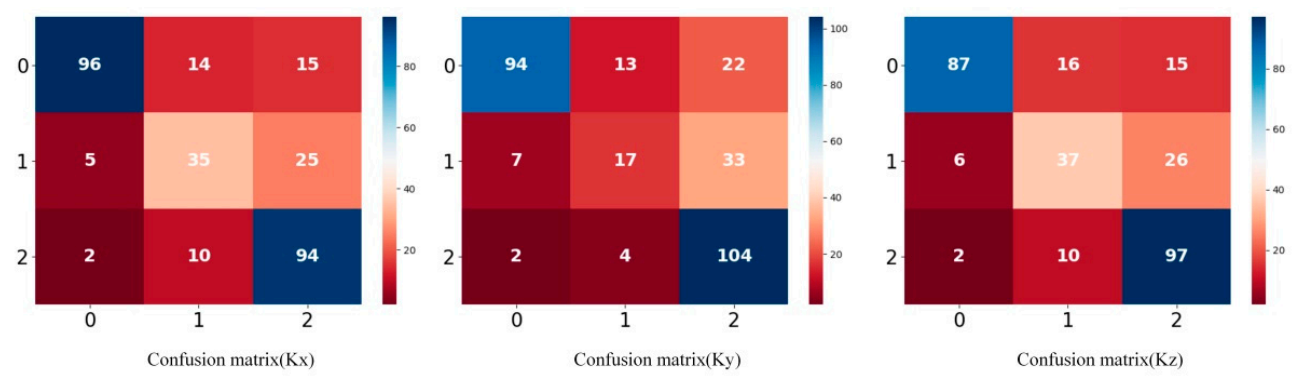

Figure 10. Confusion matrix of stiffness (MAV1) (0-high stiffness, 1-middle stiffness, 2-low stiffness).

In Figure 9, Compared with the KNN algorithm, the accuracy of stiffness classification with XGBoost is improved. The classification accuracy is over 75\% when the MAV1 is selected as the feature of sEMG signals. According to the confusion matrix of stiffness in Figure 10, the classification effect at the human arm endpoint for low stiffness and high stiffness is better than that of middle stiffness, which is similar to the results of KNN method.

\subsection{Stiffness Mapping in Contact Space}

According to the stiffness classification from the sEMG signals, the classified stiffness at the human arm endpoint can be obtained in real time, which can be mapped to naturally control the stiffness of the slave robot in contact space.

The overall block diagram of stiffness mapping for teleoperation is shown in Figure 11. In contact space, the CyberForce without force feedback and TrignoTM Wireless system are utilized simultaneously to teleoperate the slave manipulator. The feedback force is not applied to the human arm because it influents the stiffness of human arm. The Cartesian impedance controller in which the stiffness parameters can be adjusted is designed in the slave robot to execute the compliant tasks. The reference position $\mathbf{P}_{\text {end }}$ of the impedance controller is provided by the human arm motion measured by CyberForce. Besides, the classified stiffness at the human arm endpoint $\boldsymbol{K}_{\text {end }}$ can be used as the reference stiffness of the Cartesian impedance controller to regulate the compliant behaviors of the slave robot.

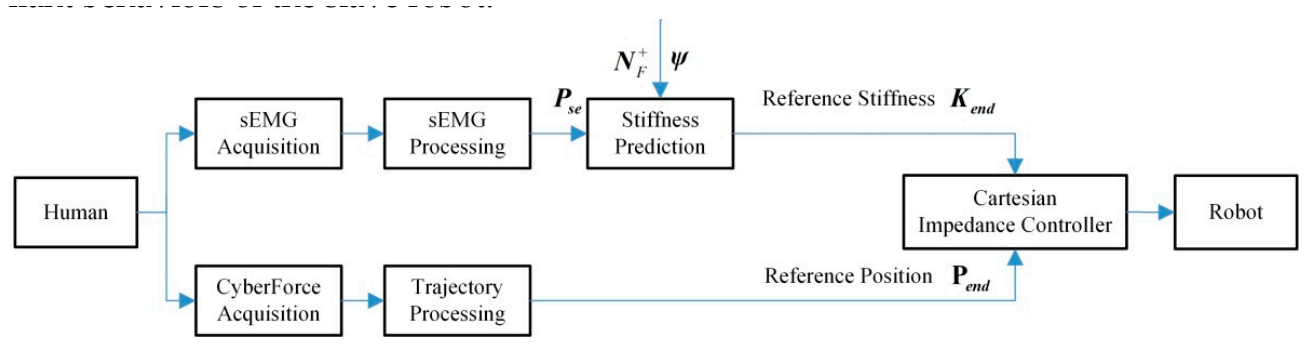

Figure 11. The overall block diagram of stiffness mapping for teleoperation.

\section{Results}

According to different workspace of robot, three different teleoperation experiments are specially designed to evaluate the effectiveness of the proposed method. In free space, the trajectory tracking experiment is utilized to evaluate the fixed mapping method. In fine space, the experiment of approaching \& grasping a drill with obstacle avoidance is accomplished to verify the virtual impedance mapping. In contact space, the experiments of human robot interaction with low and high stiffness are designed to validate to stiffness mapping method.

\subsection{Performance Evaluation for Position Teleoperation in Free Space}

The trajectory tracking experiment in free space is designed to evaluate the effectiveness of the fixed scale mapping method for position teleoperation. For all seven joints, the desired angle and 
actual angle for each joint are almost coincident, which shows that the slave manipulator tracks the master well with the fixed scale mapping, shown in Figure 12.
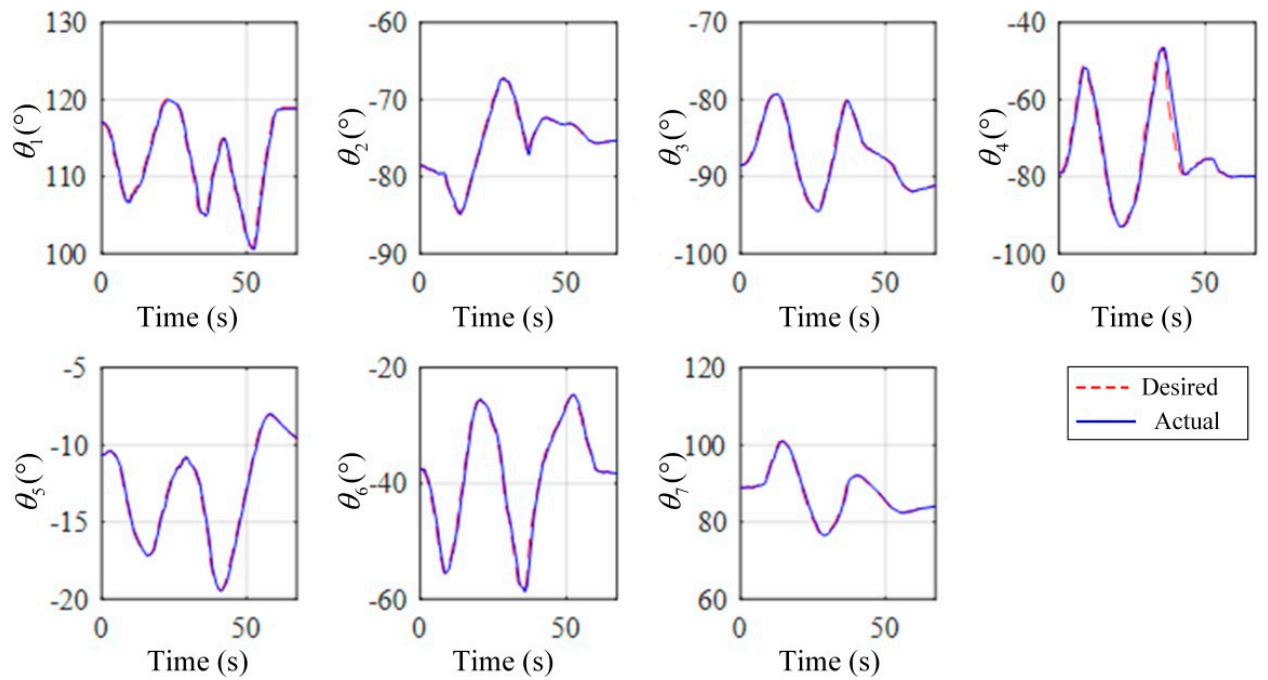

Figure 12. Trajectory tracking results with fixed scale mapping.

\subsection{Performance Evaluation for Position Teleoperation in Fine Space}

In order to verify the virtual impedance mapping in fine space, the experiment of approaching \& grasping a drill with obstacle avoidance is accomplished, shown in Figure 13. The actual robot system can obtain the obstacle information from the prior knowledge or vision of the head. To guarantee the consistency between the master and slave, a corresponding virtual obstacle is established at the master side and is utilized to implement the virtual impedance mapping. The Cartesian trajectory of manipulator is shown in Figure 14. It can be seen that the manipulator moves from the start area, avoids an obstacle in the obstacle area, and stops at the end area. In the obstacle area, since the manipulator needs to avoid the obstacle, the Cartesian trajectory of manipulator forms a convex shape. In the start and end areas, the Cartesian trajectory of manipulator is not smooth because the operator attempts to adjust the appropriate orientation at the human arm endpoint with CyberForce. It is a user related phenomenon because different operator has different ability to align the orientation. Figure 15 shows the joint trajectory of manipulator during the actual grasping process with virtual impedance mapping.

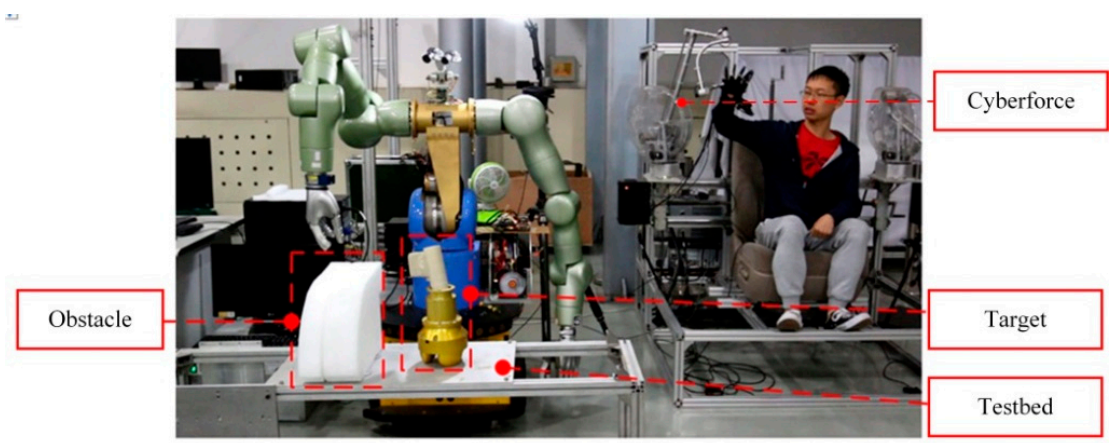

Figure 13. Experiment setup for fine space. 


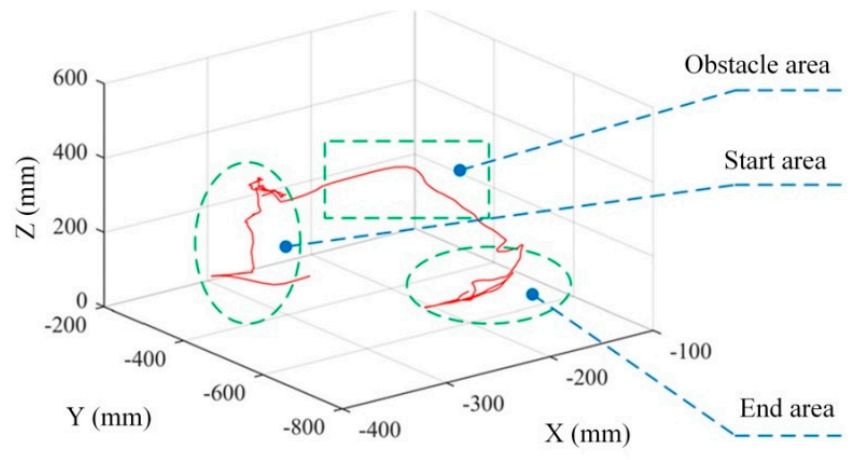

Figure 14. Cartesian trajectory of manipulator.
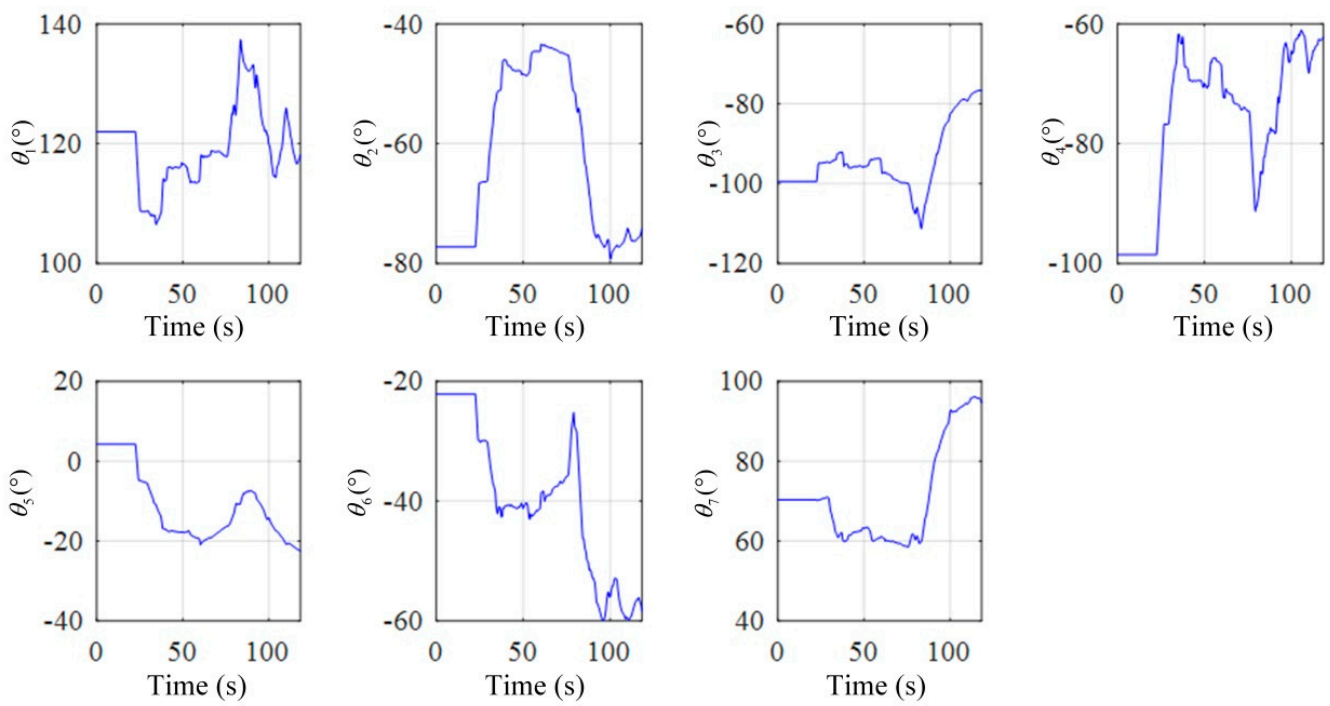

Figure 15. Actual joint trajectory with virtual impedance mapping.

\subsection{Performance Evaluation for Compliant Teleoperation in Contact Space}

In order to validate the effectiveness of stiffness mapping method for compliant teleoperation, the human robot interaction experiments with low and high stiffness are designed. In the low stiffness experiment, the operator $A$ uses the palm open gesture to generate low stiffness at his arm endpoint to teleoperate the low stiffness of slave manipulator, and the operator B pushes the manipulator with small force by his fingertip, shown in Figure 16a. Similarly, in the high stiffness experiment, the operator A uses the palm close gesture to generate high stiffness at his arm endpoint to teleoperate the high stiffness of slave manipulator, and the operator B should exert large force through his palm to push the manipulator, shown in Figure 16b.

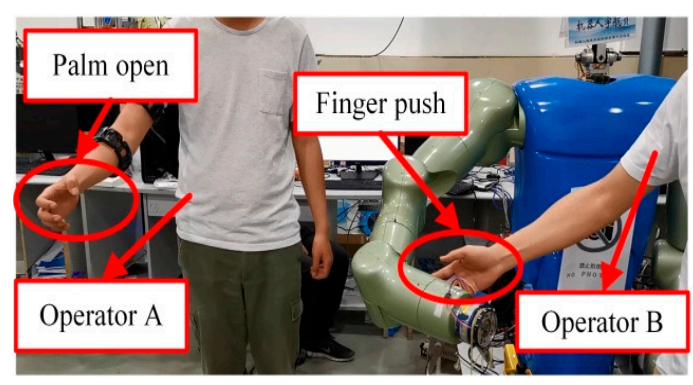

(a) Human robot interaction with low stiffness.

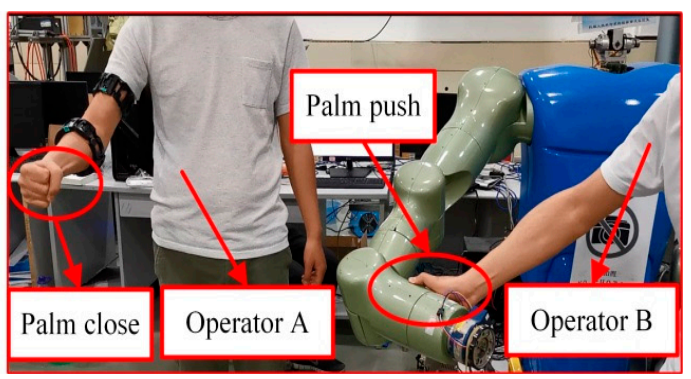

(b) Human robot interaction with high stiffness.

Figure 16. Experimental setup of human robot interaction. 
The experimental results of torque and stiffness are shown in Figure 17. In the initial stage, the interaction force is small and the stiffness of the joint is low. When the interaction force increases, the joint stiffness increases. In the experiments, the operator A can intuitively teleoperate the stiffness of the slave manipulator in order to naturally interact with the operator B.

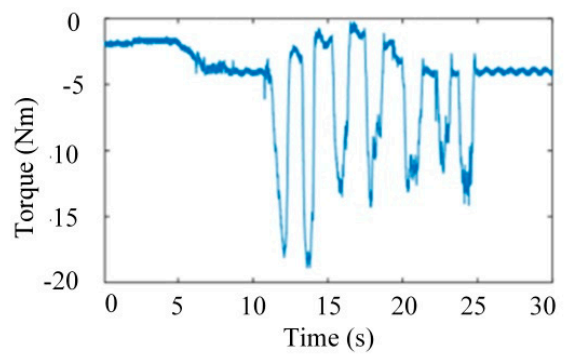

(a) Torque of Joint 4

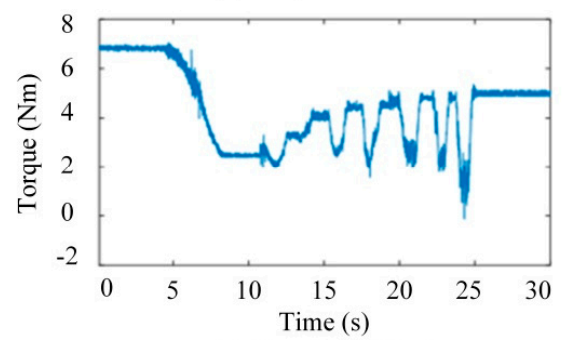

(c) Torque of Joint 5

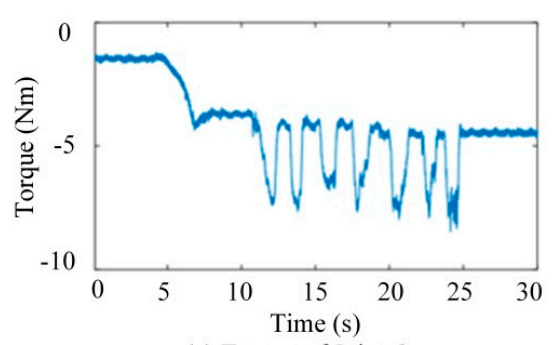

(e) Torque of Joint 6

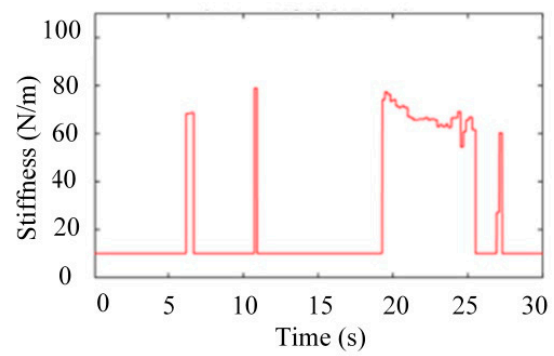

(b) Stiffness of Joint 4

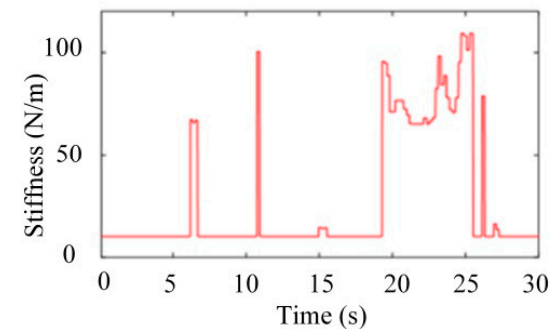

(d) Stiffness of Joint 5

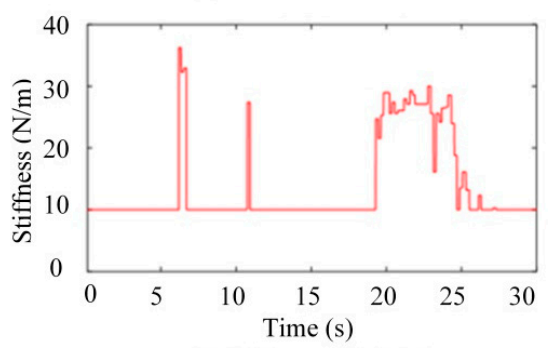

(f) Stiffness of Joint 6

Figure 17. Experimental results of torque and stiffness.

\section{Conclusions}

Humans have the ability to actively regulate their arm position and impedance to obtain a versatile and stable behavior while interacting with dynamic uncertain environments, and transferring the versatile skills of human behavior to teleoperate manipulators is a major concern in robotics. This paper proposes a hybrid mapping method with position and stiffness for manipulator teleoperation through the exoskeleton master of CyberForce and sEMG sensors. Firstly, the robot workspace is redefined into the free space, fine space and contact space. Then, the fixed scale mapping in free space and virtual impedance mapping in fine space are proposed for position teleoperation. Moreover, the stiffness at the human arm endpoint is predicted by sEMG signals, and the stiffness mapping method is utilized to adjust the stiffness of the slave manipulator. Finally, three typical experiments of trajectory tracking in free space, obstacle avoidance in fine space and human robot interaction in contact space are accomplished, and the experimental results show the effectiveness of the proposed method.

Author Contributions: Conceptualization, Z.J. and F.Y.; Funding acquisition, Z.J. and H.L.; Software, C.L. and F.Y.; Supervision, F.N.; Writing-original draft, Z.J.; Writing-review \& editing, F.N. and D.Y.

Funding: This research was funded by the National Natural Science Foundation of China, grant number 91848202 and grant number 61803124, and the Post-Doctor Research Startup Foundation of Heilongjiang Province.

Conflicts of Interest: The authors declare no conflict of interest. 


\section{References}

1. Artigas, J.; Hirzinger, G. A brief history of DLR's space telerobotics and force feedback teleoperation. Acta Polytech. Hung. 2016, 13, 239-249.

2. Ahlstrom, T.; Curtis, A.; Diftler, M.; Berka, R.; Joyce, C.; Badger, J.; Yayathi, S. Robonaut 2 on the international space station: Status update and preparations for IVA mobility. In Proceedings of the AIAA SPACE Conference and Exposition, San Diego, CA, USA, 10-12 September 2013.

3. Saltaren, R.; Barroso, A.R.; Yakrangi, O. Robotics for seabed teleoperation: Part-1-conception and practical implementation of a hybrid seabed robot. IEEE Access 2018, 6, 60559-60569. [CrossRef]

4. Mohareri, O.; Schneider, C.; Salcudean, S. Bimanual telerobotic surgery with asymmetric force feedback: A daVinci surgical system implementation. In Proceedings of the IEEE International Conference on Intelligent Robots and Systems, Chicago, USA, 14-18 September 2014; pp. 4272-4277.

5. Marturi, N.; Rastegarpanah, A.; Takahashi, C.; Adjigble, M.; Stolkin, R.; Zurek, S.; Kopicki, M.; Talha, M.; Kuo, J.A.; Bekiroglu, Y. Towards advanced robotic manipulation for nuclear decommissioning: A pilot study on tele-operation and autonomy. In Proceedings of the IEEE International Conference on Robotics and Automation for Humanitarian Applications (RAHA), Kerala, India, 18-20 December 2016; pp. 1-8.

6. Imaida, T.; Yokokohji, Y.; Doi, T.; Oda, M.; Yoshikawa, T. Ground-space bilateral teleoperation of ETS-VII robot arm by direct bilateral coupling under 7-s time delay condition. IEEE Trans. Robot. Autom. 2004, 20, 499-511. [CrossRef]

7. Niemeyer, G.; Slotine, J.E. Telemanipulation with Time Delays. Int. J. Robot. Res. 2004, 23, 873-890. [CrossRef]

8. Dolan, J.M.; Friedman, M.B.; Nagurka, M.L. Dynamic and loaded impedance components in the maintenance of human arm posture. IEEE Trans. Syst. Man Cybern. 1993, 23, 698-709. [CrossRef]

9. Castellini, C.; van der Smagt, P. Surface EMG in advanced hand prothetics. Biol. Cybern. 2009, 100, 35-47. [CrossRef] [PubMed]

10. Howard, M.; Braun, D.J.; Vijayakumar, S. Constraint-based equilibrium and stiffness control of variable stiffness actuators. In Proceedings of the International Conference on Robotics and Automation, Shanghai, China, 9-13 May 2011; pp. 5554-5560.

11. Osu, R.; Franklin, D.W.; Kato, H.; Gomi, H.; Domen, K.; Yoshioka, T.; Kawato, M. Short- and long-term changes in joint co-contraction associated with motor learning as revealed from surface EMG. J. Neurophysiol. 2002, 88, 991-1004. [CrossRef] [PubMed]

12. Hogan, N. Stable execution of contact tasks using impedance control. In Proceedings of the International Conference on Robotics and Automation, Raleigh, NC, USA, 31 March-3 April 1987; pp. 1047-1054.

13. He, W.; Ouyang, Y.; Hong, J. Vibration Control of a Flexible Robotic Manipulator in the Presence of Input Deadzone. IEEE Trans. Ind. Inform. 2017, 13, 48-59. [CrossRef]

14. Tsumugiwa, T.; Yokogawa, R.; Hara, K. Variable impedance control based on estimation of human arm stiffness for human-robot cooperative calligraphic task. In Proceedings of the International Conference on Robotics and Automation, Washington, USA, 11-15 May 2002; pp. 644-650.

15. Erden, M.S.; Billard, A. Hand Impedance Measurements during Interactive Manual Welding with a Robot. IEEE Trans. Robot. 2015, 31, 168-179. [CrossRef]

16. Walker, D.S.; Salisbury, J.K.; Niemeyer, G. Demonstrating the benefits of variable impedance to telerobotic task execution. In Proceedings of the International Conference on Robotics and Automation, Shanghai, China, 9-13 May 2011; pp. 1348-1353.

17. Ajoudani, A.; Tsagarakis, N.G.; Bicchi, A. Tele-impedance: Towards transferring human impedance regulation skills to robots. In Proceedings of the International Conference on Robotics and Automation, St. Paul, MN, USA, 14-18 May 2012; pp. 382-388.

18. Yang, C.; Zeng, C.; Liang, P.; Li, Z.; Li, R.; Su, C. Interface Design of a Physical Human-Robot Interaction System for Human Impedance Adaptive Skill Transfer. IEEE Trans. Autom. Sci. Eng. 2018, 15, 329-340. [CrossRef]

19. Luo, J.; Yang, C.; Su, H.; Liu, C. A robot learning method with physiological interface for teleoperation systems. Appl. Sci. 2019, 9, 2099. [CrossRef]

20. Laghi, M.; Ajoudani, A.; Catalano, M.G.; Bicchi, A. Tele-impedance with force feedback under communication time delay. In Proceedings of the IEEE International Conference on Intelligent Robots and Systems, Vancouver, BC, Canada, 24-28 September 2017; pp. 2564-2571. 
21. Haptic Workstation. Available online: http://www.cyberglovesystems.com/haptic-workstation (accessed on 8 September 2019).

22. Trigno Avanti Platform. Available online: https://www.delsys.com/trigno/ (accessed on 10 September 2019).

23. Cao, B.; Gu, Y.; Sun, K.; Jin, M.; Liu, H. Development of HIT humanoid robot. In Proceedings of the International Conference on Intelligent Robotics and Applications, Wuhan, China, 16-18 August 2017; pp. 286-297.

24. Li, C.; Jiang, Z.; Wang, Y.; Chen, J.; Liu, H.; Cai, H. Teleoperation of upper-body humanoid robot platform with hybrid motion mapping strategy. In Proceedings of the IEEE International Conference on Real-time Computing and Robotics (RCAR), Kandima, Maldives, 1-5 August 2018; pp. 651-656.

25. Chen, T.; Guestrin, C. Xgboost: A scalable tree boosting system. In Proceedings of the International Conference on Knowledge Discovery and Data Mining, San Francisco, CA, USA, 13-17 August 2016; pp. 785-794.

(C) 2019 by the authors. Licensee MDPI, Basel, Switzerland. This article is an open access article distributed under the terms and conditions of the Creative Commons Attribution (CC BY) license (http://creativecommons.org/licenses/by/4.0/). 